\title{
Creating and testing carbon interfaces - integrating oligomeric phthalocyanines onto single walled carbon nanotubes
}

\author{
Volker Strau $\beta,{ }^{a}$ Almudena Gallego, ${ }^{b}$ Gema de la Torre, ${ }^{b}$ \\ Thomas W. Chamberlain, ${ }^{d}$ Andrei N. Khlobystov, de Tomás Torres ${ }^{\text {bc }}$ \\ and Dirk M. Guldi ${ }^{* a}$
}

Received 10th April 2014, Accepted 11th April 2014

DOI: $10.1039 / c 4 f d 00063 c$

Two oligomers, that is, para-phenylenevinylene with either pendant $\mathrm{Pd}(॥) \mathrm{Pc}$ or $\mathrm{Zn}(\|) \mathrm{Pc}$ units, have been synthesized and their interactions with SWCNTs and peapods C60@SWCNT - have been probed by complementary spectroscopy and microscopy. Spectroscopy assisted mainly in shedding light onto electronic interactions in the ground and excited state, while microscopy enabled insights into the degree of individualizing SWCNTs/C 60 @SWCNTs and their stabilization.

\section{Introduction}

Nanosized carbon based materials offer exciting opportunities in a wide palette of areas of nanotechnology, since they possess novel electrical, magnetic, thermal, optical, mechanical, chemical, and morphological properties. ${ }^{\mathbf{1 , 2}}$ Carbon nanotubes (CNTs) are cylinders constituted of one - single walled carbon nanotubes (SWCNTs) - or more - multiwalled carbon nanotubes (MWCNTs) - graphene sheets with diameters from 0.4 to $5 \mathrm{~nm}$ and from 1.4 to $200 \mathrm{~nm}$, respectively, and lengths up to half-meter. ${ }^{3}$ In the past two decades, CNTs have triggered considerable attention owing to their unprecedented and interesting features. The band gap and optical properties of semiconducting CNTs are widely tunable with their diameters enabling specific light absorption. ${ }^{4}$ The latter span from the ultraviolet to the infrared region. More notable are the electronic properties: CNTs reveal

\footnotetext{
${ }^{a}$ Department of Chemistry and Pharmacy Interdisciplinary Center for Molecular Materials (ICMM) Friedrich-Alexander-Universitaet Erlangen-Nuernberg, Egerlandstr. 3, 91058, Erlangen, Germany. E-mail: dirk.guldi@fau.de

${ }^{b}$ Departamento de Química Orgánica, Universidad Autónoma de Madrid, Cantoblanco, 28049, Madrid, Spain 'Instituto Madrileño de Estudios Avanzados (IMDEA)-Nanociencia, c/ Faraday 9, Cantoblanco, 28049, Madrid, Spain

${ }^{d}$ School of Chemistry, University of Nottingham, Nottingham, NG7 2RD, UK

${ }^{e}$ Nottingham Nanoscience \& Nanotechnology Centre, University of Nottingham, University Park, Nottingham, $N G 72 R D, U K$
} 
ballistic charge conduction, field effect mobilities on the order of $10^{5} \mathrm{~cm}^{2} \mathrm{~V}^{-1} \mathrm{~s}^{-1}$ at room temperature, electrical conductivities up to $1.7 \times 10^{6} \mathrm{~S} \mathrm{~cm}^{-1}$ and current carrying capability or ampacity up to $10^{9} \mathrm{~A} \mathrm{~cm}^{-2} .^{5}$

In SWCNT bundles, fluorescence is hardly ever observed. Metallic SWCNTs, which are statistically present in bundles, are the inception for photoexcited carriers in semiconducting SWCNTs to relax along nonradiative pathways. ${ }^{6}$ On the contrary, fluorescence measurements with individual SWCNTs have revealed distinct fluorescing transitions for more than 30 different SWCNTs.

The combination of SWCNTs with electron donors or acceptors generates active materials, which can produce electrical energy when irradiated. ${ }^{7}$ In contrast to empty fullerenes and endohedral metallofullerenes, SWCNTs are, however, very elusive species when it comes to the characterization of their metastable states. This problem mainly arises from the polydispersive nature of SWCNT samples and the inevitable presence of SWCNTs in bundles of different sizes. An interesting aspect for electronic applications is the manipulation of the electronic properties of semiconducting SWCNTs, which is at the forefront of current investigations. ${ }^{8}$ Charge transfer/doping is a leading example of a potential strategy toward this aim, which is expected to substantially increase the density of free charge carriers and thereby enhance the electrical and thermal conductivities. Notable are contributions in the area of field effect transistors. ${ }^{9}$ Along the same lines, the effects that are generated when replacing carbon by boron, nitrogen or silicon are carefully studied in terms of transport properties of SWCNTs. A final example of charge transfer interactions includes a tunable photosensor. An alternative approach rests on the meta-stable doping of SWCNTs, triggered by light. In particular, the use of photoexcited state electron donors or electron acceptors generate transient charge carriers in the conduction and valence bands of SWCNTs, respectively. One way to achieve this goal relies on the use of versatile protocols to covalently attach electron donors or electron acceptors to SWCNTs. ${ }^{10}$ However, the impact that the covalent functionalization exerts on the electronic structure is noticeable and affects transport properties, etc. Thus, efforts are directed to a non-covalent strategy, that is, the implementation of multifunctional groups without compromising the electronic and structural features of SWCNTs.

What renders working with SWCNTs so difficult is that all methods used to synthesize them in reasonable quantities produce SWCNTs with a very broad range of electronic, thermal, and structural properties that change depending on the different kinds. ${ }^{11}$ A viable strategy implements charge transfer/doping. Our approach, on one hand, facilitates the integration of a molecular handle, while, on the other hand, preserving the SWCNT original electronic structure. Such interactions implicitly require the physical adsorption of suitable molecules onto the SWCNT sidewalls. We have performed systematic and complementary investigations by means of microscopy and spectroscopy, which have shed light onto mutual interactions between semiconducting SWCNTs and our dye-containing surfactants, towards the realization of two major milestones. The first milestone is the establishment of a versatile methodology to achieve watersoluble SWCNTs for processing them under environmentally friendly conditions. ${ }^{12,13}$ In fact, microscopy demonstrated the benefits of tightly interacting $\pi$ systems towards the successful debundling and suspension. The second milestone is the establishment of a protocol to achieve $p$-doped SWCNTs for the 


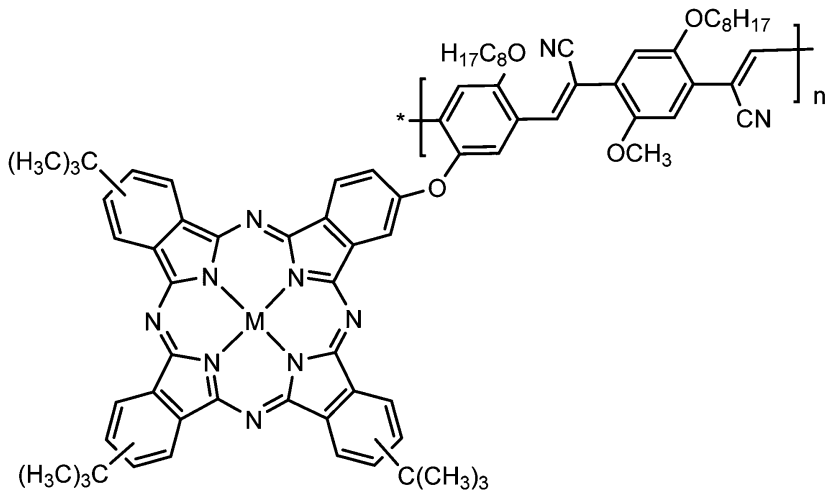

1a $M=Z n(I I)$

1b $M=P d(I I)$

Scheme 1 PPV-Zn(॥)Pc 1a and PPV-Pd(॥)Pc 1b.

integration into novel electronic devices. Enhancing the $p$-type character is also achieved by trapping $\mathrm{C}_{60}$ inside of SWCNT $-\mathrm{C}_{60} @$ SWCNT. ${ }^{14}$ In fact, preliminary experiments point to the successful shift of electron density from SWCNT to $\mathrm{C}_{60}{ }^{15}$

Oligomers as templates for electroactive components are similarly susceptible towards chemical changes. Simply incorporating electron withdrawing functionalities such as cyano groups into the oligomer backbone will help to transform the oligomer to feature $n$-type instead of $p$-type character. ${ }^{16}$ These experiments should be contrasted to work on electron donating polyethylene imines, which transform $p$-type SWCNT into $n$-type SWCNT. ${ }^{17}$

In this contribution, we opted for two phthalocyanine (Pc)-containing oligomers, that is, PPV-Zn(II)Pc 1a and PPV-Pd(II)Pc 1b (Scheme 1), as excited state electron donors and immobilize them onto SWCNTs and $\mathrm{C}_{60} @$ @WCNTs. $\mathrm{C}_{60} @$ @WCNTs were chosen owing to the intrinsic charge transfer from SWCNTs to encapsulated $\mathrm{C}_{60}$ as a means to facilitate immobilizing PPV-Zn(II)Pc 1a and PPV$\operatorname{Pd}(\mathrm{II}) \mathrm{Pc} 1 \mathrm{~b}$, on one hand, and individualizing not only $\mathrm{C}_{60} @$ SWCNTs but also SWCNTs, on the other hand.

\section{Experimental section}

\section{General}

Chemicals were purchased from Sigma Aldrich and used as received without further purification. IR spectra were recorded with a Bruker Vector 22 spectrophotometer. MALDI-TOF MS and HRMS spectra were recorded with a Bruker Reflex III. NMR spectra were recorded with a Bruker AC-300 instrument. Column chromatography was carried out on silica gel Merck-60 (230-400 mesh, $60 \AA$ ) and TLC on aluminum sheets pre-coated with silica gel 60 F254 (E. Merck).

\section{9,16,23-Tri-tert-butyl-2-[4-(2-ethyl)hexyloxy-2,5-bis(hydroxymethyl)phenoxy] phthalocyaninato palladium(II) (mixture of regioisomers) (3)}

A mixture of $4\left[\left(4^{\prime}\right.\right.$-(2-ethyl)hexyloxy-2' ${ }^{\prime} 5^{\prime}$-bis(methylacetoxy)]phenoxyphthalonitrile (2) $)^{18}$ (200 mg, $\left.0.41 \mathrm{mmol}\right)$, 4-tert-butyphthalonitrile (227 mg, $1.23 \mathrm{mmol}$ ) and 
DBU $(0.08 \mathrm{~mL})$ in $N, N$-dimethylethanolamine $(4 \mathrm{~mL})$ was heated at $100{ }^{\circ} \mathrm{C}$ under argon atmosphere until complete dissolution of the materials. Then, $\mathrm{PdCl}_{2}$ (95 $\mathrm{mg}, 0.54 \mathrm{mmol}$ ) was added and the mixture was refluxed for $20 \mathrm{~h}$. After cooling, methanol $(10 \mathrm{~mL})$ was added and the mixture was refluxed again for further $2 \mathrm{~h}$ and poured into water $(30 \mathrm{~mL})$. The resulting dark blue solid was filtrated and washed with water, mixtures of methanol-water $(1: 2)$ and $(1: 1)$, and methanol. Afterwards, the solid was dried and redissolved in tetrahydrofuran and purified by column chromatography $\left(\mathrm{SiO}_{2}\right)$ using $\mathrm{CHCl}_{3}$-hexane-dioxane $(1: 2: 0.1)$ as eluent. Phthalocyanine 3 was obtained as a blue solid $(79 \mathrm{mg}, 18 \%) . \delta_{\mathrm{H}}(500 \mathrm{MHz}$; $\left.\mathrm{CDCl}_{3} ; \mathrm{Me}_{4} \mathrm{Si}\right)$ 0.5-2.0 (42H, m, CH, $\left.\mathrm{CH}_{2}, \mathrm{CH}_{3}\right), 4.2\left(2 \mathrm{H}, \mathrm{d}, \mathrm{ArOCH}_{2}\right)$, 5.1-5.6 $(4 \mathrm{H}$, $\left.\mathrm{m}, \mathrm{ArCH}_{2} \mathrm{OH}\right), 6.8-7.0(2 \mathrm{H}, \mathrm{m}, \mathrm{ArH}), 7.7-8.2(4 \mathrm{H}, \mathrm{m}, \mathrm{Pc}-\mathrm{H})$ and 8,6-9.2 (8 H, m, $\mathrm{Pc}-\mathrm{H}) ; \mathrm{m} / z$ (MALDI, DCTB) 1074-1066. $\left(\mathrm{C}_{60} \mathrm{H}_{64} \mathrm{~N}_{8} \mathrm{O}_{4} \mathrm{Pd}\right.$ requires 1066). $\lambda_{\max }$ (tetrahydrofuran)/nm $(\log \varepsilon) 332$ (3.8), 596 (3.7) and 660 (4.5). FTIR spectrum shows the corresponding $\mathrm{O}-\mathrm{H}$ wide band at $3410 \mathrm{~cm}^{-1}$.

\section{9,16,23-Tri-tert-butyl-2-[4-(2-ethyl)hexyloxy-2,5-diformyl)phenoxy] phthalocyaninato palladium(II) (mixture of regioisomers) (4)}

To a well stirred solution of 1-hydroxy-1,2-benziodoxole-3(1H)-one-1-oxide (IBX) (50 mg, $0.17 \mathrm{mmol})$ in DMSO (60 mL), compound $3(60 \mathrm{mg}, 0.056 \mathrm{mmol}$ ) was added at once. The solution was stirred at room temperature for $24 \mathrm{~h}$ and then, poured over brine $(80 \mathrm{~mL})$ and extracted with diethyl ether $(3 \times 30 \mathrm{~mL})$. The organic layer was separated, washed with a saturated solution of sodium bicarbonate $(2 \times 30 \mathrm{~mL})$, brine $(2 \times 30 \mathrm{~mL})$ and dried over sodium sulfate. After filtration of the drying agent, the solvent was evaporated and the blue solid was purified on a BiobeadsR $\odot$ column using THF as eluent, affording aldehyde 4 as dark blue solid (39 mg, 65\%). $\delta_{\mathrm{H}}\left(500 \mathrm{MHz} ; \mathrm{CDCl}_{3} ; \mathrm{Me}_{4} \mathrm{Si}\right)$ 0.9-2.1 (42 H, m, CH, $\left.\mathrm{CH}_{2}, \mathrm{CH}_{3}\right), 4.3\left(2 \mathrm{H}, \mathrm{m}, \mathrm{ArOCH}_{2}\right), 7.3-8.8(14 \mathrm{H}, \mathrm{m}, \mathrm{Pc}-\mathrm{H}, \mathrm{ArH}), 10.68(1 \mathrm{H}, \mathrm{bs}$, $\mathrm{CHO}$ ) and 11.00 ( $1 \mathrm{H}$, bs, CHO); $m / z$ (MALDI, DCTB) 1070-1062 $\left(\mathrm{C}_{60} \mathrm{H}_{60} \mathrm{~N}_{8} \mathrm{O}_{4} \mathrm{Pd}\right.$ requires 1062). $\lambda_{\max }$ (tetrahydrofuran)/nm (log $\varepsilon$ ) 331 (3.9), 595 (3.8) and 659 (4.5). FTIR spectrum reveals a strong band at $1610 \mathrm{~cm}^{-1}(\mathrm{vC}-\mathrm{O})$.

\section{Synthesis of PPV-PdPc oligomer 1b via Knoevenagel reaction}

Diformylderivative 4 (30 mg, $0.028 \mathrm{mmol}$ ), 2,5-bis(cyanomethyl)-4-(2-ethylhexyloxy)anisol, ${ }^{18}(9 \mathrm{mg}, 0.029 \mathrm{mmol})$ and anhydrous potassium tert-butoxide (5 $\mathrm{mg}, 0.04 \mathrm{mmol})$ were dissolved in a mixture of dry tetrahydrofuran $(6 \mathrm{~mL})$ and dry tert-butanol $(3 \mathrm{~mL})$. The solution was heated at $60{ }^{\circ} \mathrm{C}$ and then a solution of tetrabutylammonium hydroxide $(9 \mathrm{~mL}, 0.1 \mathrm{M}$ in methanol) was added at once, turning the solution a dark green colour. After heating for $24 \mathrm{~h}$, further anhydrous potassium tert-butoxide ( $5 \mathrm{mg}, 0.04 \mathrm{mmol}$ ) and tetrabutylammonium hydroxide solution ( $9 \mathrm{~mL}, 0.1 \mathrm{M}$ in methanol) were added. The reaction was maintained until consumption of the starting diformyl compound ( $c a .24 \mathrm{~h}$ ). Then, the solvents were evaporated and the residue was triturated in methanol $(20 \mathrm{~mL})$ and acidified with a drop of acetic acid in an ultrasound bath. The solid obtained was purified on a BiobeadsR@ column, with dichloromethane as solvent. The main fraction (first to elute) was separated and the solvent eliminated. After washing with a mixture of hexane-acetone $(1: 1)$ the green compound was dried in vacuum affording $16 \mathrm{mg}$ of 3 as a green solid. $\delta_{\mathrm{H}}\left(500 \mathrm{MHz}\right.$; THF- $\left.\mathrm{d}_{8} ; \mathrm{Me}_{4} \mathrm{Si}\right)$ 0.6-2.3 (m, CH, $\left.\mathrm{CH}_{2}, \mathrm{CH}_{3}, \mathrm{C}\left(\mathrm{CH}_{3}\right)_{3}\right)$, 5.0-5.3 (2× m, $\left.\mathrm{ArOCH}_{2}, \mathrm{ArOCH}_{3}\right)$, 8.0-8.2 (m, ArH, 
vinylH), 9.0-9.5 (m, Pc-H). $\lambda_{\max }$ (tetrahydrofuran)/nm (log $\left.\varepsilon\right) 327$ (4.2), 600 (3.7) and 661 (4.2). FTIR spectrum shows a band at $2231 \mathrm{~cm}^{-1}(v \mathrm{C}-\mathrm{N})$.

\section{Synthesis of $\mathrm{C}_{60} @$ @WWCNTs}

$\mathrm{C}_{60} @ S W C N T s$ were produced from arc discharge SWCNT (Helix Material Solutions Inc., USA ) and $\mathrm{C}_{60}$ (SES Research, USA). As received SWCNTs (20 mg) were annealed in air at $370{ }^{\circ} \mathrm{C}$ for $10 \mathrm{~min}$ to remove the end caps and residual amorphous carbon from the nanotube interior, and then stored at $300{ }^{\circ} \mathrm{C}$ to avoid the presence of water in the sample. A 3-fold excess of $\mathrm{C}_{60}(60 \mathrm{mg})$ was added to the nanotubes and the resultant material was sealed in a quartz tube under reduced pressure $\left(6 \times 10^{-6} \mathrm{mbar}\right)$ and heated at $550^{\circ} \mathrm{C}$ for 3 days. The material was then washed with $\mathrm{CS}_{2}(20 \mathrm{~mL})$ to remove any fullerenes adsorbed on the surface of the nanotubes and filtered through a PTFE membrane (pore diameter $=0.2 \mu \mathrm{m}$ ).

\section{Spectroscopy and Microscopy}

Steady state absorption measurements were carried out with either a Lambda 2 UV/Vis/NIR-spectrometer (Perkin Elmer) or a Cary 5000 UV/Vis/NIR-spectrometer (Varian). Steady state fluorescence measurements were performed with a FluoroMax®-3 spectrofluorometer (Horiba). All spectra were corrected for the instrument response. Transmission electron microscopy was performed with a TEM 912 Omega (Zeiss) at an acceleration voltage of $80 \mathrm{kV}$. Femtosecond transient absorption studies were carried out with a CPA 2101 (Clark-MXR Inc.) coupled to a Helios transient absorption pump/probe system (Ultrafast systems) with $387 \mathrm{~nm}$ laser pulses ( $1 \mathrm{kHz}, 150 \mathrm{fs}$ pulse width). Raman spectra were recorded using a FTRaman RFS 100 system (Bruker) with a Ge detector using a 1064 nm Nd-YAG laser for excitation. High resolution transmission electron microscopy analysis was performed using a JEOL-2100F field-emission gun microscope. The imaging conditions were carefully tuned by lowering the accelerating voltage of the microscope to $100 \mathrm{kV}$ and reducing the beam current density to a minimum. Nanotube samples (2-3 mg) were dispersed in $2 \mathrm{~mL}$ methanol using an ultrasonic bath and deposited onto Lacey carbon-film-coated TEM copper grids. All experiments were performed at room temperature in either solid state or in dimethylformamide (DMF) spectrophotometric grade (99.8\%, Sigma-Aldrich). CoMoCAT® SWCNTs SG 65, enriched with $(6,5)$-SWCNTs were obtained from SIGMA ALDRICH (Purity $\geq 77 \%$ carbon as SWCNT, diameter $0.7-0.9 \mathrm{~nm},>90 \%$ semi-conducting).

\section{Results and Discussions}

\section{Synthesis}

The synthesis of the $o$-PPV oligomer with pendant Pd(II)Pc units (1b) by Knoevenagel condensation procedures requires the preparation of the corresponding diformyl- and dicianomethylene-functionalized precursors (Scheme 2). On one hand, the synthesis of the Pc-containing co-monomer was carried out by initial mixed condensation reaction between phthalonitrile $2^{18}$ and 4-tert-butylphthalonitrile, followed by hydrolysis of the acetate moieties to hydroxymethyl groups to afford 3 and further oxidation with IBX to give the diformyl compound 4. On the other hand, the synthesis of the dicyanomethylene co-monomer 5 was 


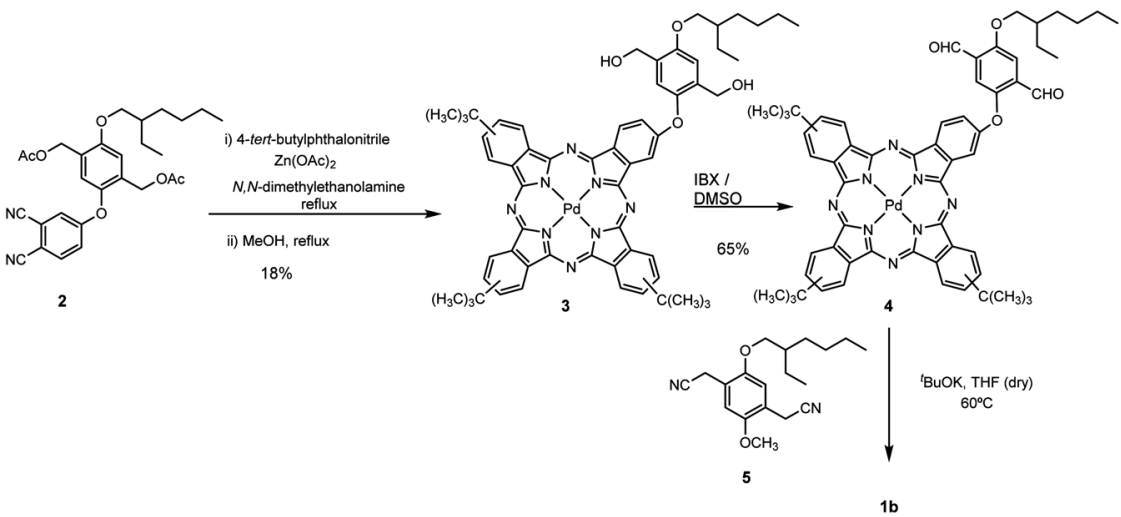

Scheme 2 Synthesis of Pd(॥)Pc-containing oligomer - PPV-Pd(॥)Pc (1b)

carried out as previously described. ${ }^{19,20}$ Polymerization of co-monomers 3 and 4 was accomplished following similar conditions to that previously reported for related $\mathrm{Zn}$ (II)Pc-containing $o$ PPV oligomers 1a, that is, condensation in the presence of potassium tert-butoxide and tetrabutylammonium hydroxide during long reaction times $(48 \mathrm{~h})$.

1b is very soluble in polar solvents such as THF and DMF. FT-IR showed a characteristic peak at $2231 \mathrm{~cm}^{-1}$, which is assigned to the cyano moieties. ${ }^{1} \mathrm{H}-$ NMR in THF- $\mathrm{d}_{8}$ showed broad signals for the aromatic and also for the $\mathrm{CH}_{3} \mathrm{O}$ and $-\mathrm{CH}_{2} \mathrm{O}$ protons.

\section{Photophysical and microscopic characterization}

When compared to the known PPV-Zn(II)Pc 1a, both the absorption and the fluorescence features of PPV-Pd(II)Pc 1b are considerably blue shifted by $14 \mathrm{~nm}$ (Fig. 1). In particular, the Q-bands shift from 676 to $662 \mathrm{~nm}$ and from 686 to 672 $\mathrm{nm}$. At first glance, the fluorescence patterns of the two oligomers are equivalent. A closer look reveals, however, that the fluorescence quantum yield is - due to an increased spin orbit coupling - significantly reduced to $0.5 \%$ in PPV-Pd(II)Pc 1 b when compared to PPV-Zn(II)Pc 1a. For example, photoexcitation into its Soretband at $350 \mathrm{~nm}$ fails to produce any appreciable fluorescence. Only upon direct excitation into the Q-band fluorescence is observed.

Recently, the interactions of PPV-Zn(II)Pc 1a with SWCNTs were tested with HiPco SWCNTs. ${ }^{16}$ In the current work we used two other samples of SWCNTs, that is, CoMoCAT SWCNTs and $\mathrm{C}_{60} @$ @WWNTs. The first are enriched with semiconducting SWCNTs to a degree of up to 90\%. Typically, CoMoCAT SWCNTs exhibit very low defect rates and are - due to their small diameter of $\sim 0.8 \mathrm{~nm}-$ comparatively flexible. On the other hand, $\mathrm{C}_{60} @$ @WCNTs have larger diameters of $\sim 1.4 \mathrm{~nm}$ and the incorporation of $\mathrm{C}_{60}$ adds some degree of stiffness.

For the production of $1 \mathbf{b} / \mathrm{SWCNT}$ and $1 \mathrm{a} / \mathrm{SWCNT}$ hybrids we followed a sequential enrichment procedure. To this end, we started with a $1 \times 10^{-5} \mathrm{M}$ solution of the respective oligomers to disperse maximum amounts of SWCNTs/ $\mathrm{C}_{60} @ S W C N T s$. A decolorization, which sets in as soon as the mixture was stirred, points to rather strong interactions between the oligomers and SWCNTs or $\mathrm{C}_{60} @ S W C N T s$. To avoid the latter, SWCNTs/C ${ }_{60} @ S W C N T s$ were pre-dispersed in 

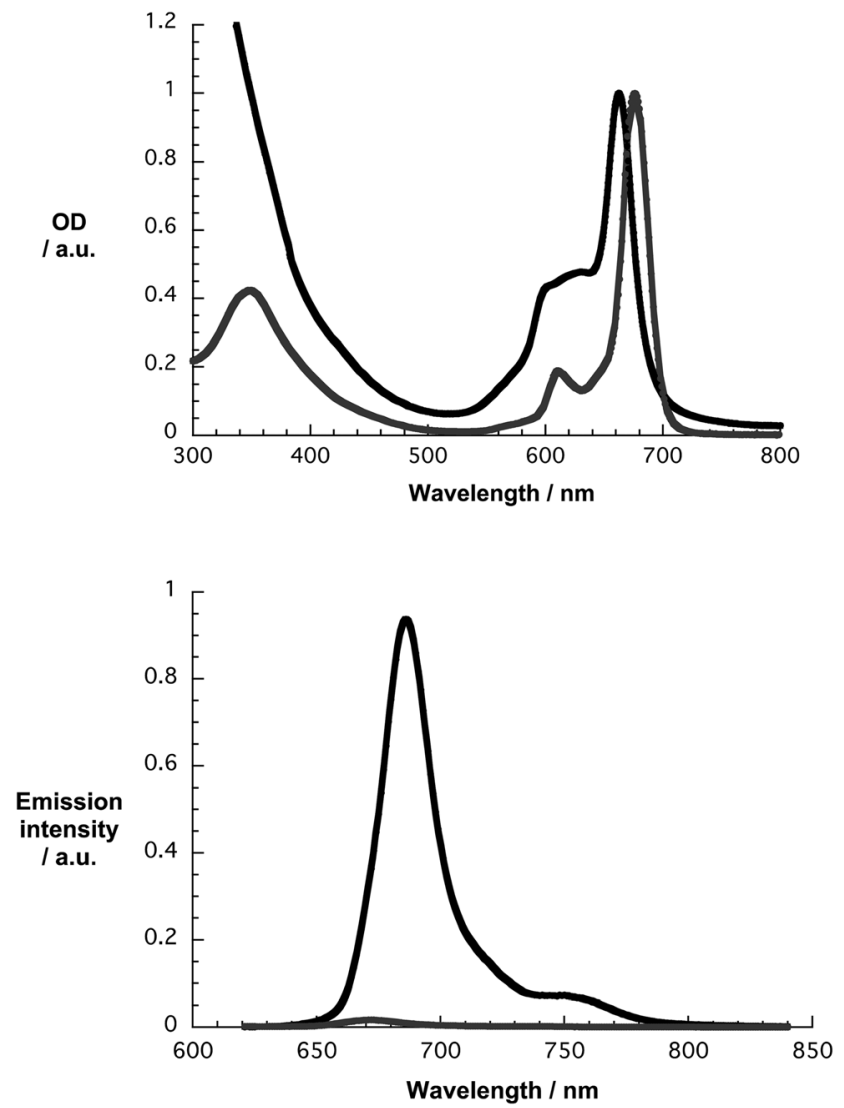

Fig. 1 Upper part - normalized absorption spectra of $1 \times 10^{-5} \mathrm{M}$ solutions of PPV-Pd(॥)Pc 1b oligomer (black spectrum) and PPV-Zn(॥)Pc 1a oligomer (dark grey spectrum) in DMF at room temperature. Lower part - fluorescence spectra of $1 \times 10^{-5} \mathrm{M}$ solutions of PPVPd(॥)Pc 1b oligomer (dark grey spectrum) and PPV-Zn(॥)Pc 1a oligomer (black spectrum) in DMF at room temperature upon photoexcitation at $600 \mathrm{~nm}$.

a bare minimum of DMF. The pre-dispersed SWCNTs/ $\mathrm{C}_{60} @ S W C N T s$ were added stepwise to the oligomer solutions followed by $10 \mathrm{~min}$ of ultrasonication. Steady state absorption and fluorescence spectroscopy were employed to monitor the progress of the enrichment. The steps were repeated until no further changes were observed in the optical spectra (Fig. 2).

Firstly, the typical SWCNT transitions emerge in the near infrared parts of the absorption spectra. Upon applying prolonged ultrasonications, these bands sharpen and blue-shift. As a matter of fact, we consider this as an indicator for debundling of SWCNTs. Secondly, upon adding SWCNTs/ $\mathrm{C}_{60} @$ SWCNTs to either of the oligomer solutions, a sequential decrease of the Q-band absorptions is discernable with the concomitant formation of new absorption bands at 698 and $684 \mathrm{~nm}$ for the $\mathrm{Zn}$ (II)Pc and Pd(II)Pc, respectively. From previous studies, we infer that this band is due a charge-transfer state. ${ }^{16,21,22}$

A look at the fluorescence spectra reveals that the successive addition of SWCNTs to solutions of either PPV-Zn(II)Pc 1a or PPV-Pd(II)Pc 1b induces a 

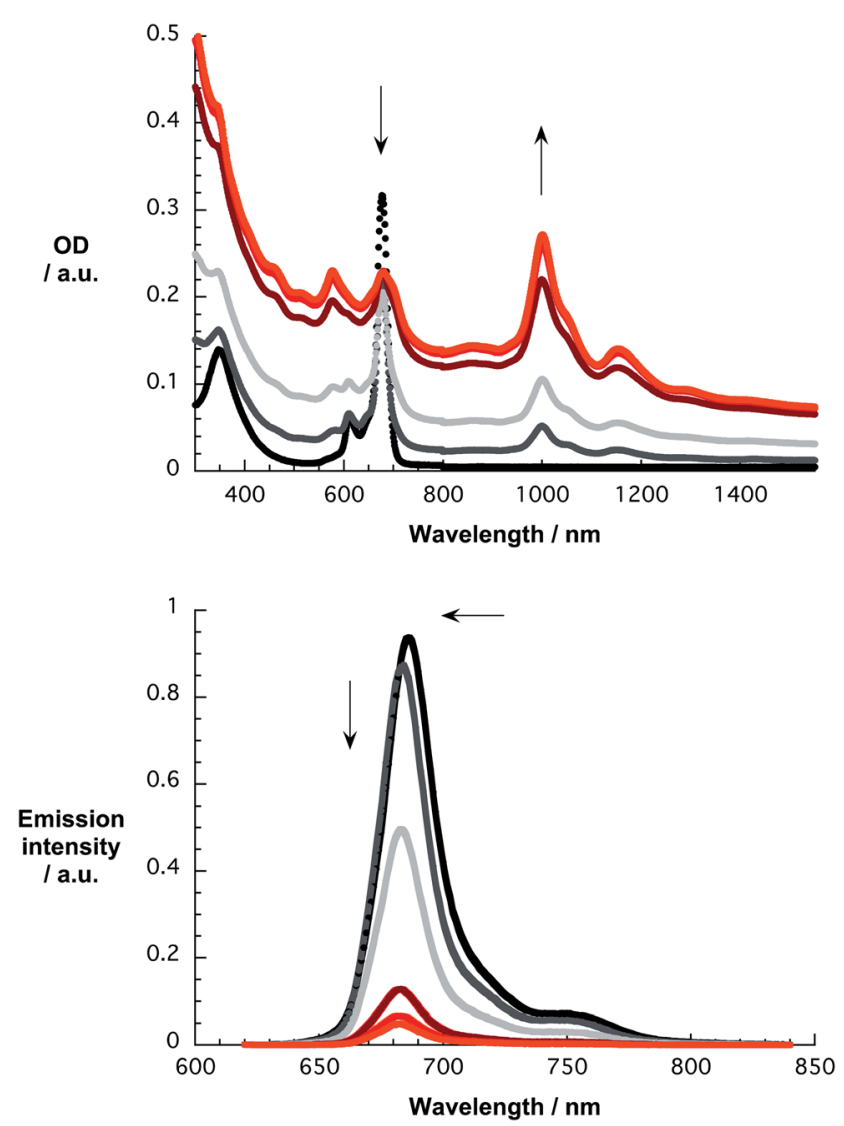

Fig. 2 Upper part - absorption spectra recorded during the sequential enrichment of a 1 $\times 10^{-5}$ M PPV-Zn(॥)Pc 1a solution with CoMoCAT SWCNTs in DMF at room temperature. Lower part - fluorescence spectra recorded during the sequential enrichment of a $1 \times$ $10^{-5} \mathrm{M} 1 \mathrm{l}$ solution with COMoCAT SWCNTs in DMF at room temperature upon photoexcitation at $600 \mathrm{~nm}$.

significant quenching of the phthalocyanine related fluorescence. From the latter we deduce the formation of stable charge transfer hybrids. Nevertheless, we cannot rule out the presence of aggregates of $\mathbf{1 a}$ or $\mathbf{1 b}$, in which the phthalocyanines stack. To this end, interactions with SWCNTs and $\mathrm{C}_{60} @$ SWCNTs induce the break up of the aggregates.

Likewise, the enrichment procedure with $\mathrm{C}_{60}$ @SWCNTs resulted in the same trends as noted for CoMoCAT SWCNTs. Nevertheless, the absorption features are drastically different (Fig. 3). $\mathrm{C}_{60} @ S W C N T$, for instance, shows two broad sets of absorptions, namely one at $\sim 1000 \mathrm{~nm}$ and one at $\sim 1800 \mathrm{~nm} .{ }^{23}$ The latter relates to $\mathrm{S}_{11}$ transitions, while the earlier is assigned to the corresponding $\mathrm{S}_{22}$ transitions. ${ }^{20}$ Striking is the intensity of the charge transfer band at $\sim 700 \mathrm{~nm}$, which develops and intensifies upon sequential addition of $\mathrm{C}_{60}$ @SWCNTs. Overall, the rather weak and broad nature of the $\mathrm{C}_{60} @$ @WCNT centered absorptions point to poorly individualized $\mathrm{C}_{60} @$ @WWCNTs. We hypothesize that the stiffness and the diameter of $\mathrm{C}_{60} @$ @WCNTs compared to CoMoCAT SWCNTs might prevent a better 


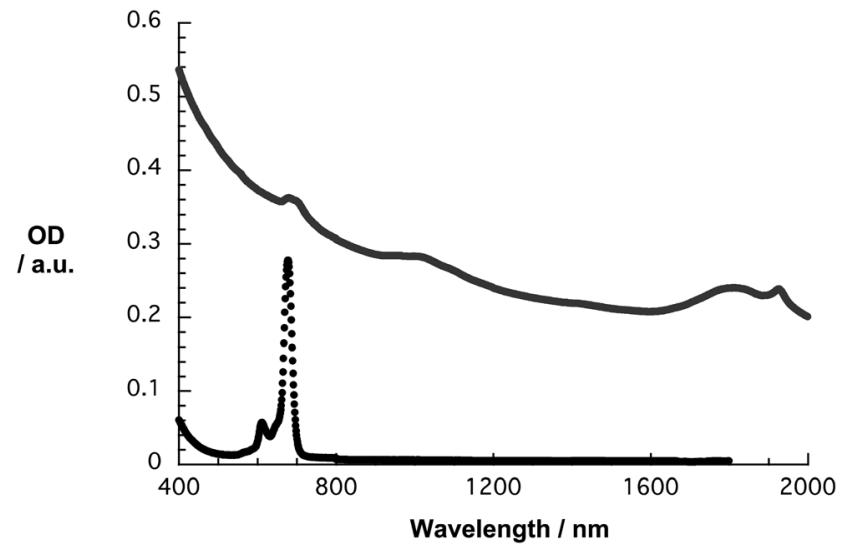

Fig. 3 Absorption spectra recorded before (black spectrum) and after (dark grey spectrum) the sequential enrichment of a $1 \times 10^{-5} \mathrm{M}$ PPV-Zn(॥)PC 1a solution with $\mathrm{C}_{60}$ aSWCNTs in DMF at room temperature.

individualization. Comparative absorption and fluorescence titration assays with predispersed $\mathrm{C}_{60} @$ @WWCNTs in DMF and small amounts of, for example, solid 1b corroborate the aforementioned. Representative absorption spectra for $\mathrm{C}_{60} @$ @SWCNTs and $\mathbf{1 b}$ are shown in Fig. 4. Following the addition of first amounts of $\mathbf{1 b}$ the charge transfer feature at $684 \mathrm{~nm}$ is discernable. In the presence of additional amounts of $\mathrm{Pd}(\mathrm{II}) \mathrm{Pc}$, the $662 \mathrm{~nm}$ absorption of free $\mathrm{Pd}(\mathrm{II}) \mathrm{Pc}$ is noted and the overall ratio between the two absorptions, that is $684 \mathrm{~nm}$ absorption versus $662 \mathrm{~nm}$ absorption, reflects the equilibration in the complex formation. In line with the strong fluorescence quenching, which was observed during the above described enrichment process, the increase in $\mathrm{Pd}(\mathrm{II}) \mathrm{Pc}$ fluorescence is barely detectable throughout the course of the titration.

To gather additional information on the electronic interactions we performed complementary Raman spectroscopic analyses. The Raman specimen were

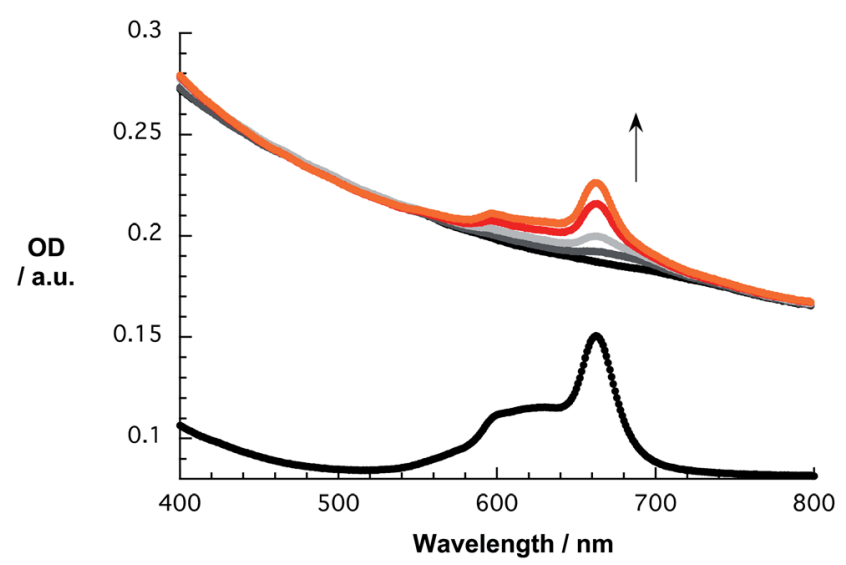

Fig. 4 Titration assays of $\mathrm{C}_{60}$ (aSWCNTs with PPV-Pd(॥)Pc 1b in DMF at room temperature. 
prepared by drop-casting the hybrid suspensions onto gold coated object slides and the solvent was evaporated under reduced pressure. All spectra were recorded upon excitation at $1064 \mathrm{~nm}$, baseline corrected, and normalized with respect to the $\mathrm{G}^{+}$-band. Comparison of the spectra before and after functionalization revealed a slight down-shift of the respective $\mathrm{G}^{+}$-bands. It is known from doping experiments with SWCNTs that shifting of RBM and $\mathrm{G}^{+}$-modes indicate charge transfer effects. ${ }^{24}$ In the present case, the shifts towards smaller frequencies denote a shift of charge density from 1a to SWCNTs.

When turning to the radial breathing modes (RBM), further insights into possible electronic interactions are gathered. In Fig. 5, the normalized RBM

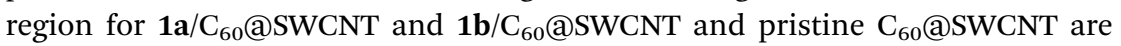
shown. Importantly, the RBM mode at $167 \mathrm{~cm}^{-1}$ correlates with that of $\mathrm{C}_{60}$ @SWCNTs featuring diameters of $\sim 1.4 \mathrm{~nm}$ as, for instance, in $(16,3)$ or $(14,6)$ SWCNTs. ${ }^{25}$ Importantly, the RBM undergoes a slight shift towards higher
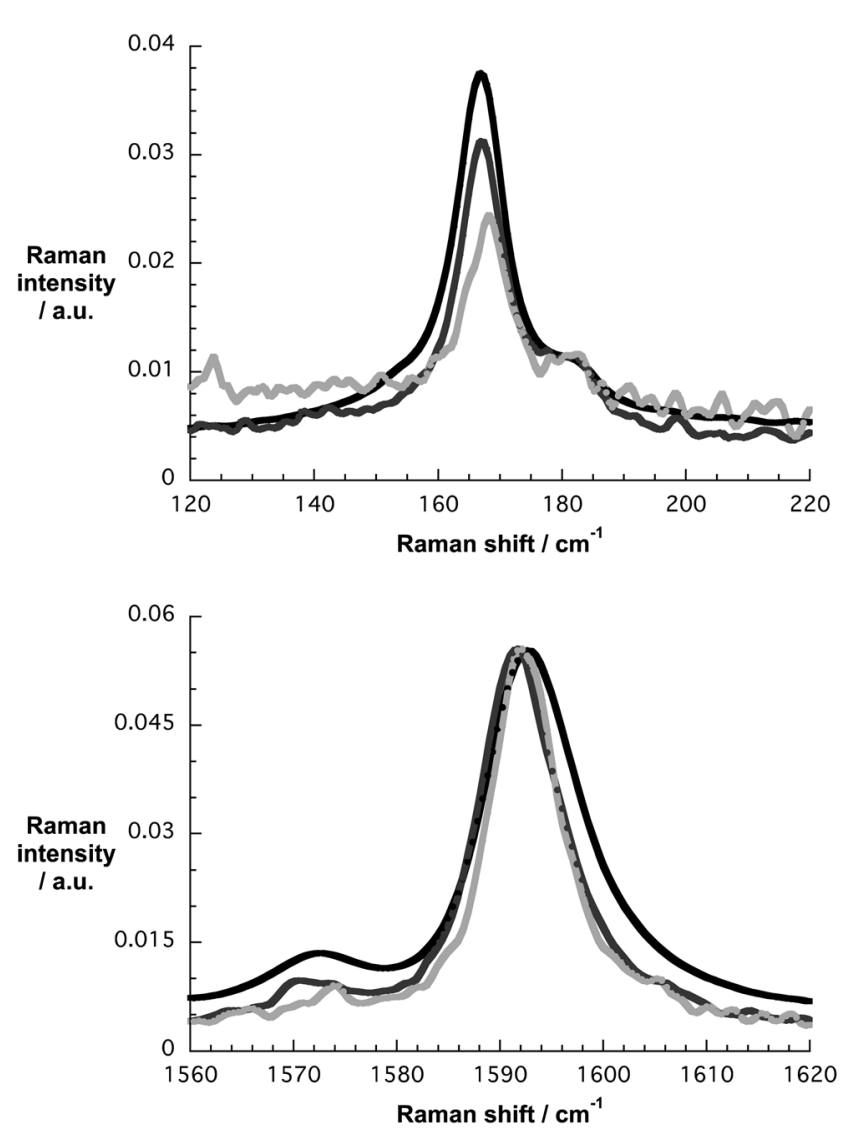

Fig. 5 Upper part - normalized solid state Raman spectra $\left(\lambda_{\text {ex }}=1064 \mathrm{~nm}\right)$ of pure $\mathrm{C}_{60}$ @SWCNT (black spectrum), 1b/C 60 @SWCNT (dark grey spectrum), and $1 \mathrm{a} /$ $\mathrm{C}_{60}$ @SWCNT (light grey spectrum) with particular emphasis on the RBM region. Lower part - normalized solid state Raman spectra $\left(\lambda_{\text {ex }}=1064 \mathrm{~nm}\right.$ ) of pure $\mathrm{C}_{60}$ @SWCNT (dark

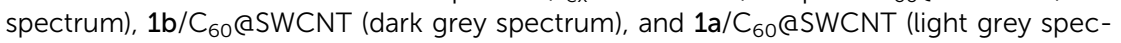
trum) with particular emphasis on the G-band region. 


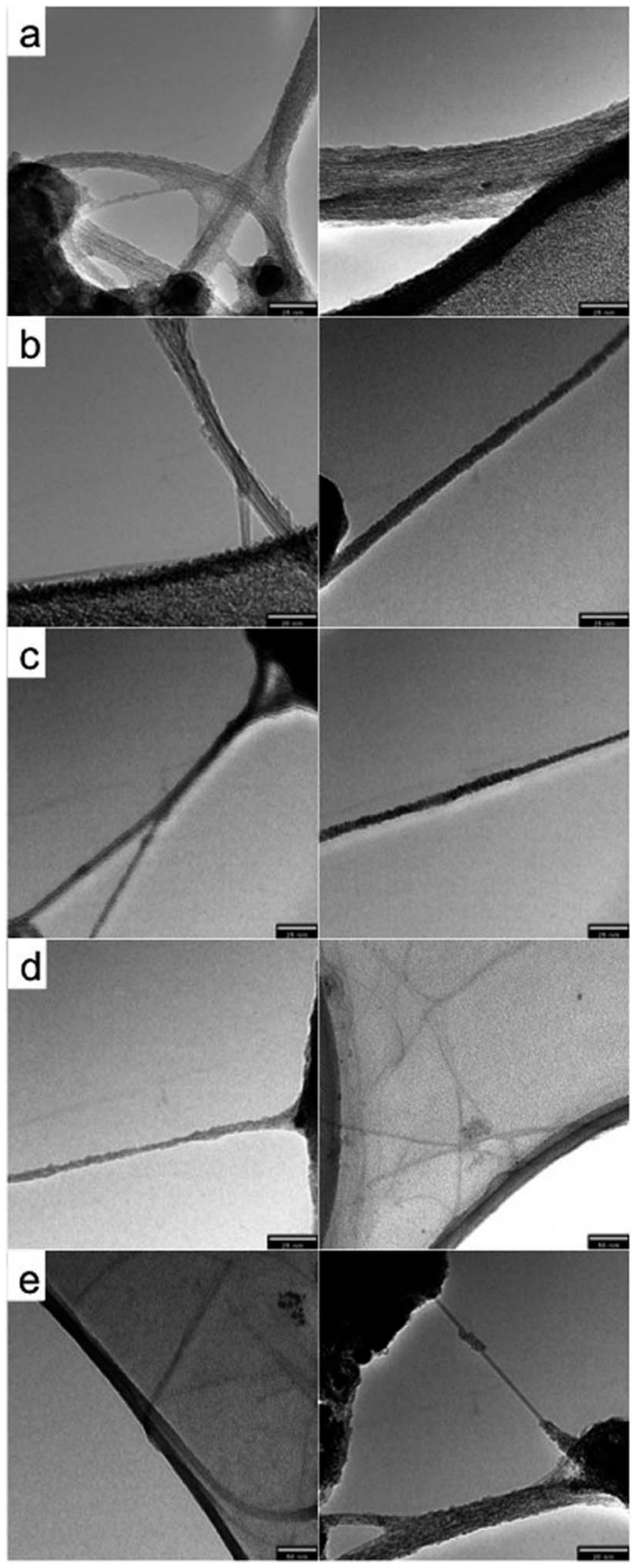

Fig. 6 a) TEM images of $C_{60}$ aSWCNT bundles. Scale bars are $25 \mathrm{~nm}$. b) TEM images of a small bundle of partially wrapped $1 \mathrm{~b} / \mathrm{C}_{60}$ @SWCNT on the left and a fully PPV-Pd(॥)Pc $1 \mathrm{~b}$ functionalized $C_{60}$ @SWCNT on the right. Scale bars are 20 and $25 \mathrm{~nm}$. c) TEM images of a small bundle and an individualized $1 \mathrm{~b} /$ SWCNT on the left and right. respectively. Scale bars are $25 \mathrm{~nm}$. d) TEM images of an individualized 1a/SWCNT on the left and a corresponding overview image on the right. Scale bars are 25 and $50 \mathrm{~nm}$. e) TEM images of an individualized partially wrapped $1 \mathrm{a} / \mathrm{C}_{60}$ @SWCNT on the left and a corresponding overview image on the right. Scale bars are 50 and $20 \mathrm{~nm}$. 

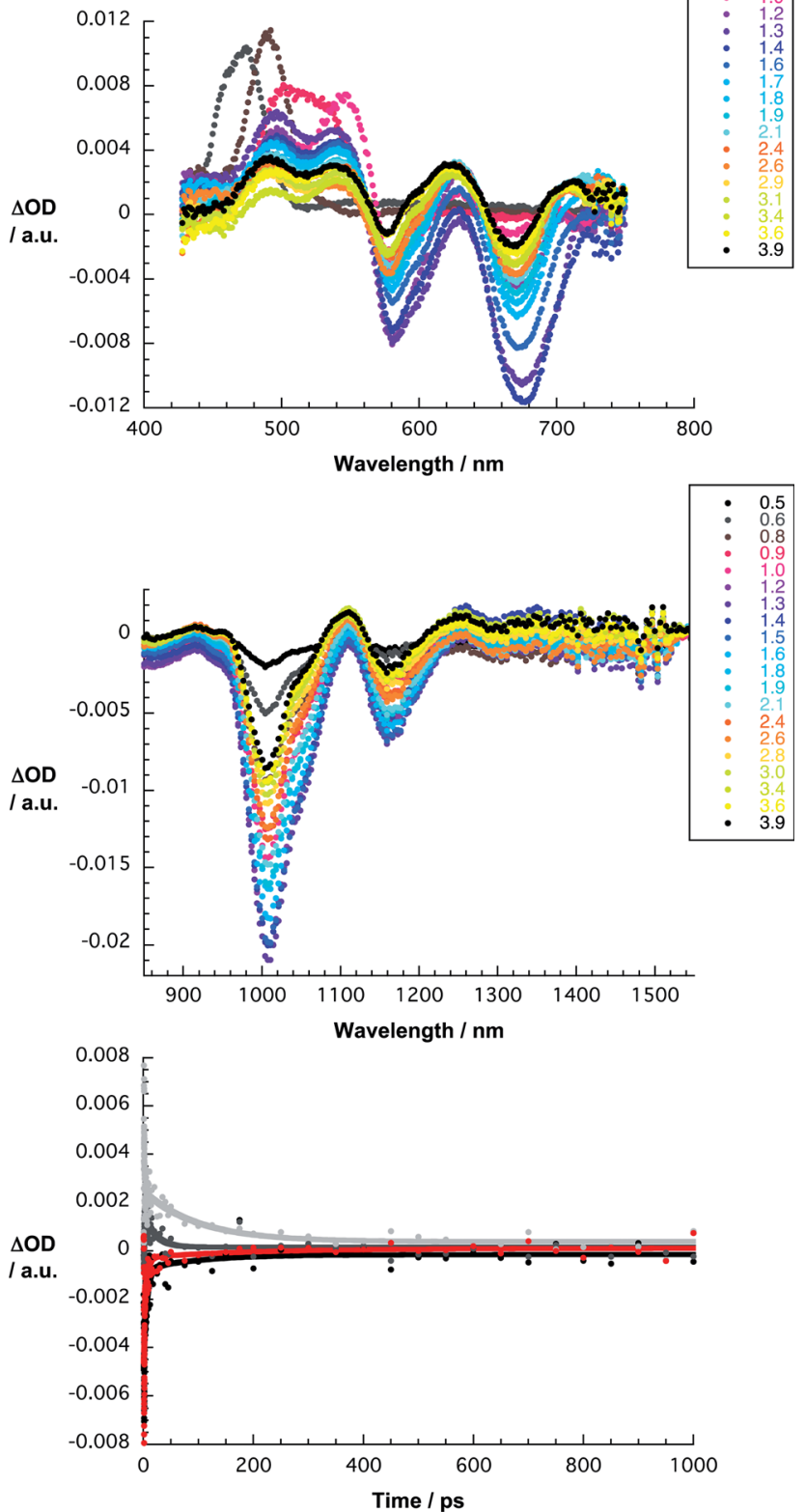

Fig. 7 Upper part - differential absorption spectra (visible) obtained upon femtosecond pump probe experiments $(387 \mathrm{~nm})$ of $1 \mathrm{~b} / \mathrm{SWCNTs}$ in DMF with several time delays between 0.5 and 3.9 ps at room temperature. Central part - differential absorption spectra (near infrared) obtained upon femtosecond pump probe experiments $(387 \mathrm{~nm}$ ) of $1 \mathrm{~b} /$ SWCNTs in DMF with several time delays between 0.5 and 3.9 ps at room temperature. Lower part - time absorption profiles of the spectra shown in the upper and central parts at 1165 (black spectrum), 1110 (dark grey spectrum), 580 (red spectrum), and $540 \mathrm{~nm}$ (light grey spectrum) monitoring the excited state decay. 
frequencies upon immobilization of the oligomers and decrease in intensity. ${ }^{\mathbf{2 6}}$ Both of these effects are typically observed in charge transfer hybrids containing SWCNTs.

Microscopic characterizations by means of transmission electron microscopy (TEM) were performed with all possible combinations of PPV-Zn(II)Pc 1a/PPV$\operatorname{Pd(II)Pc~1b~and~SWCNTs/C_{60}@SWCNTs~-~Fig.~6.~To~this~end,~dilute~solutions~of~}$ the hybrids were dropcasted onto Lacey carbon/Cu grids. The solutions were soaked by capillary forces to obtain individualized SWCNTs/ $\mathrm{C}_{60} @ S W C N T s$ crossing over the holes of the Lacey film. By this method, we are able to corroborate individual and freestanding SWCNTs/ $\mathrm{C}_{60} @$ SWCNTs, which is crucial in order to visualize the amorphous oligomer coating. ${ }^{27}$
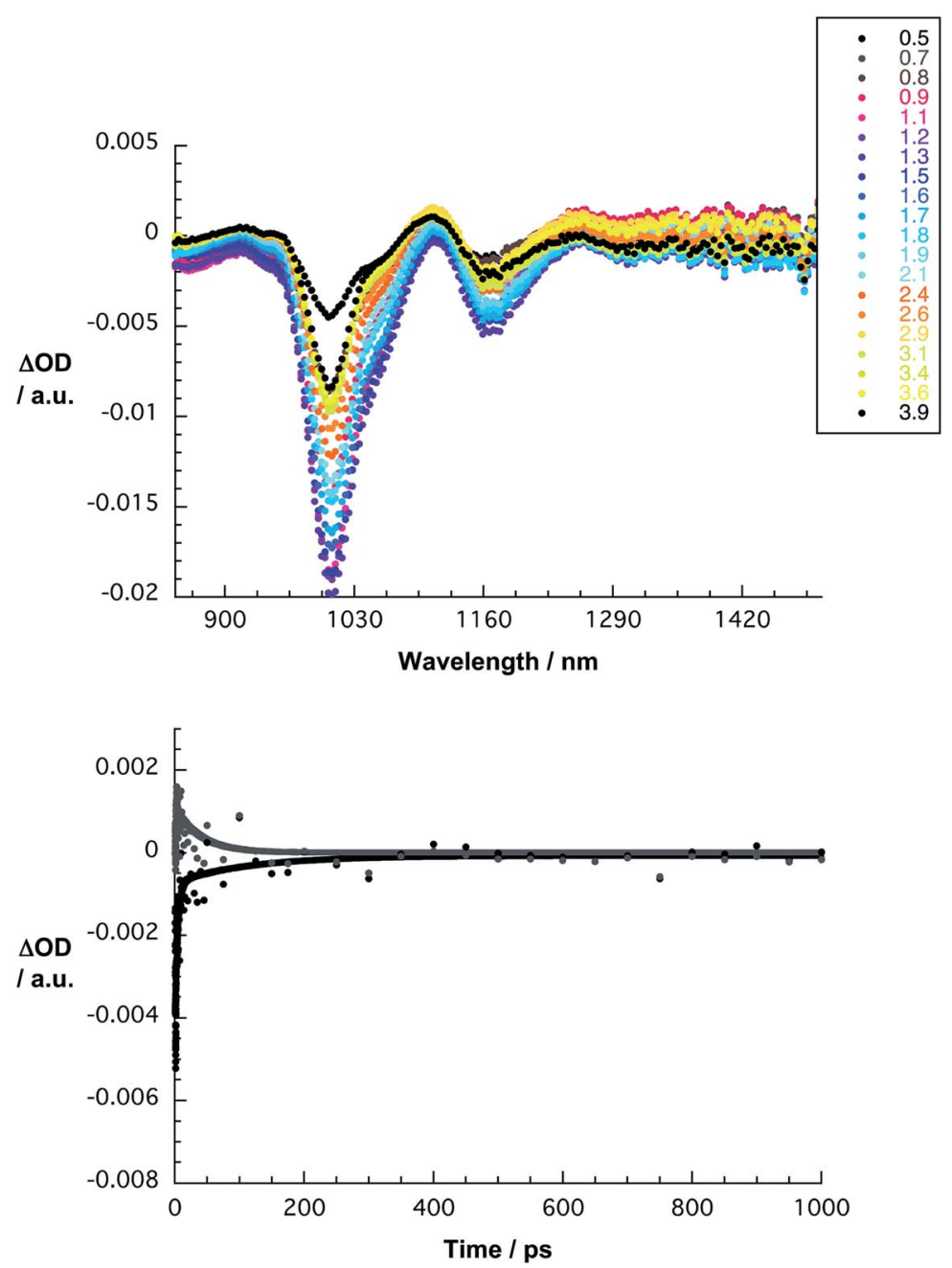

Fig. 8 Upper part - differential absorption spectra (near infrared) obtained upon femtosecond pump probe experiments $(387 \mathrm{~nm}$ ) of $1 \mathrm{a} /$ SWCNTs in DMF with several time delays between 0.5 and 3.9 ps at room temperature. Lower part - time absorption profiles of the spectra shown in the upper part at 1165 (black spectrum) and $1110 \mathrm{~nm}$ (dark grey spectrum) monitoring the excited state decay. 

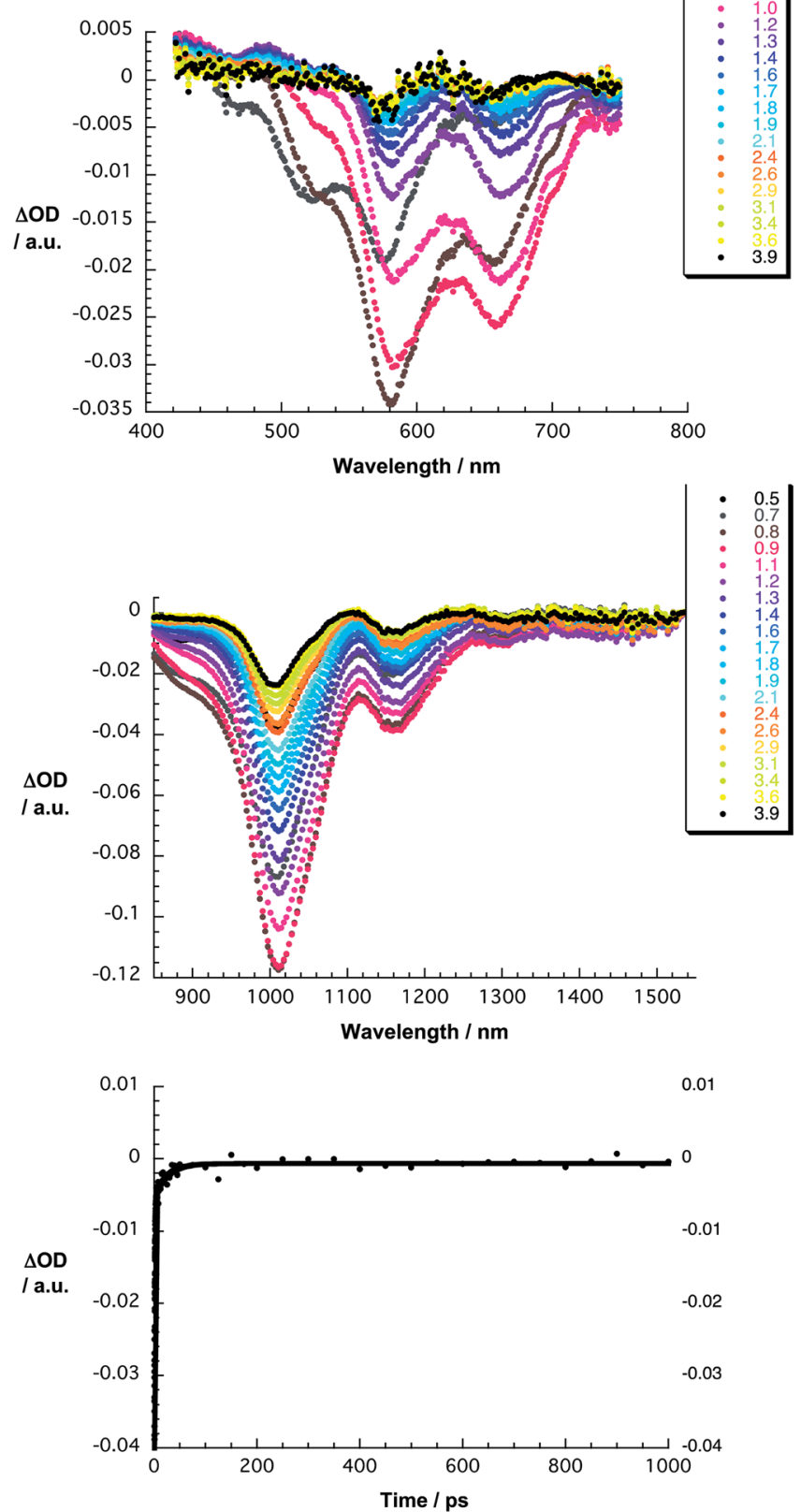

Fig. 9 Upper part - differential absorption spectra (visible) obtained upon femtosecond pump probe experiments $(387 \mathrm{~nm}$ ) of $1 \mathrm{~b} / \mathrm{SWCNT}$ thin films with several time delays between 0.5 and 3.9 ps at room temperature. Central part - differential absorption spectra (near infrared) obtained upon femtosecond pump probe experiments $(387 \mathrm{~nm}$ ) of $1 \mathrm{~b} /$ SWCNT thin films with several time delays between 0.5 and 3.9 ps at room temperature. Lower part - time absorption profile of the spectra shown in the central parts at $1159 \mathrm{~nm}$ (black spectrum) monitoring the excited state decay. 
As for the oligomer wrapped SWCNTs/ $\mathrm{C}_{60} @ S W C N T s$, throughout the examined areas predominantly small bundles of and/or loosely entangled SWCNTs/ $\mathrm{C}_{60} @ S W C N T$ were found. We commonly observe the tangling of individualized SWCNTs and smaller bundles during the preparation of TEM samples. In stark contrast only large and straighten bundles $(\sim 20-50 \mathrm{~nm})$ were seen for TEM grids prepared with non-functionalized SWCNTs/C $\mathrm{C}_{60} @ S W C N T s$. Therefore, we deduce an efficient debundling of SWCNTs/C $\mathrm{C}_{60} @$ SWCNT upon wrapping with 1a/1b. Independent confirmation for the debundling came from overview images. SWCNTs and $\mathrm{C}_{60} @$ @WCNTs on Lacey films reveal reasonable individualization for the probed hybrids. Representative images of individualized SWCNTs/ $\mathrm{C}_{60} @$ @WCNT are shown in Fig. 6. In each of the images, a relatively dense
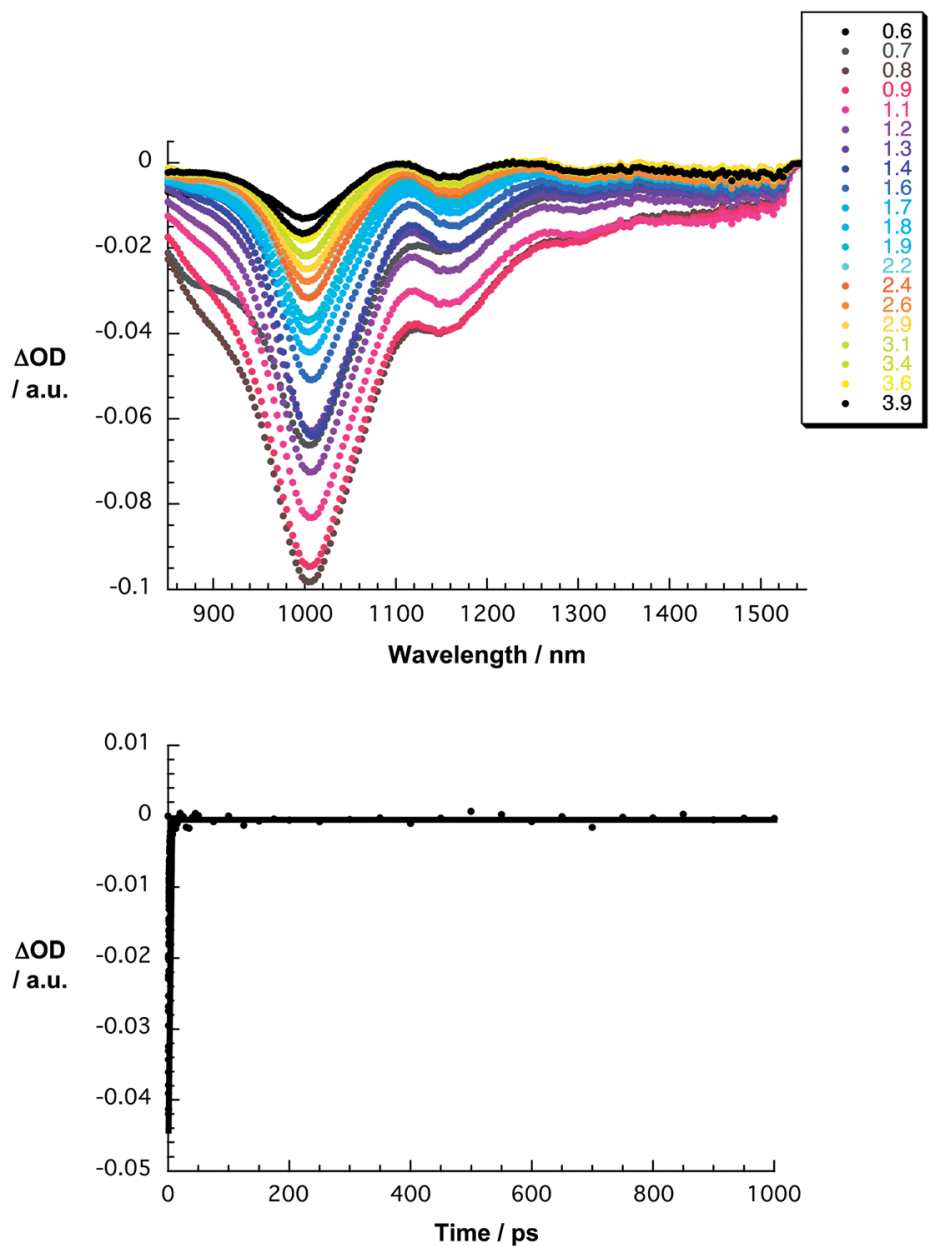

Fig. 10 Upper part - differential absorption spectra (near infrared) obtained upon femtosecond pump probe experiments $(387 \mathrm{~nm}$ ) of $1 \mathrm{a} / \mathrm{SWCNT}$ thin films with several time delays between 0.5 and 3.9 ps at room temperature. Lower part - time absorption profile of the spectra shown in the upper part at $1159 \mathrm{~nm}$ (black spectrum) monitoring the excited state decay. 
amorphous coating around SWCNTs is noted. In the case of $\mathrm{C}_{60} @$ SWCNTs, the amorphous $\mathbf{1 a} / \mathbf{1} \mathbf{b}$ coating hampers, however, the visualization of the encapsulated fullerenes. It is only in the references, that is, in the absence of $\mathbf{1 a} / \mathbf{1 b}$, that the fullerene arrays are recognizable. In the latter, well-defined and large sized bundles of $\mathrm{C}_{60} @$ @WCNTs predominate, while 1a/1 b coated $\mathrm{C}_{60} @$ @SWCNTs are rather randomly ordered.

Finally and as a complement to the aforementioned fluorescence titration experiments, we turned to pump probe experiments. For the first time, we compared solution based experiments with those performed in solid state films. As to solution based experiments, $387 \mathrm{~nm}$ photoexcitation of 1b/SWCNTs - Fig. 7
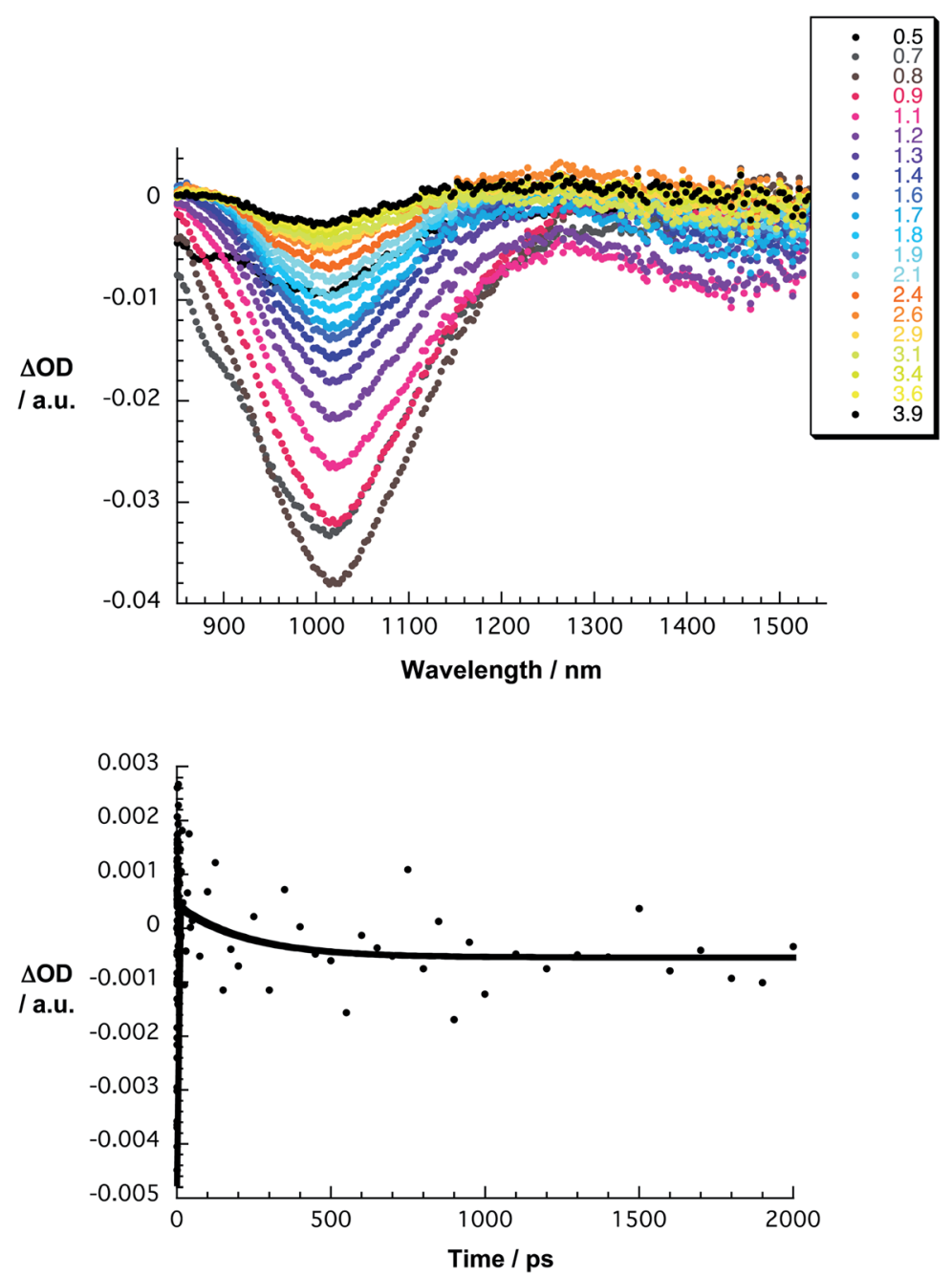

Fig. 11 Upper part - differential absorption spectra (near infrared) obtained upon femtosecond pump probe experiments $\left(387 \mathrm{~nm}\right.$ ) of $1 \mathrm{a} / \mathrm{C}_{60}$ aSWCNT thin films with several time delays between 0.5 and 3.9 ps at room temperature. Lower part - time absorption profile of the spectra shown in the upper part at $1159 \mathrm{~nm}$ (black spectrum) monitoring the excited state decay. 
- results in differential absorption changes that include maxima at 495, 542, 630, $730,1110,1256$, and $1345 \mathrm{~nm}$ as well as minima at 580, 677, 1007, and $1164 \mathrm{~nm}$. Notably, the minima reflect the ground state absorption maxima and, in turn, attest the photoexcitation of $\mathbf{1 b} /$ SWCNTs. In contrast to reference experiments with SWCNTs, whose transient features are subject to a biexponential decay but to no appreciable red or blue shifts, photoexcited 1b/SWCNTs reveal a 5 ps lasting blue shift of all the excited state characteristics. The maxima shift to $490,622,713$, 1106, 1250, and $1340 \mathrm{~nm}$, while the minima are now seen at 575, 667, 1004, and $1159 \mathrm{~nm}$. Implicit is in line with previous reports, electron injection into the conduction bands of SWCNTs and oxidation of $\mathbf{1 b}$. On the longer time scale, that is, with 135 ps, the SWCNT conduction band electrons and holes on $\mathbf{1 b}$ recombine to populate the ground state. The same findings were gathered for 1a/SWCNTs Fig. 8. For example, the 1007 and 1164 nm minima transform via 1004 and 1159 $\mathrm{nm}$ intermediates with lifetimes of 115 and $65 \mathrm{ps}$ to the ground state. ${ }^{28}$

In thin films - Fig. 9 and 10 - upon photoexciting 1b/SWCNTs 486, 624, 730, 1116, and $1273 \mathrm{~nm}$ maxima together with 582, 661, 1009, and $1161 \mathrm{~nm}$ minima were noted. Following the trend in suspensions the maxima and minima blue shift to $480,612,701,1102$, and $1244 \mathrm{~nm}$ as well as 576, 652, 1005, and $1155 \mathrm{~nm}$, respectively, before reinstating the baseline. Considering lifetimes of 46 and 25 ps, we conclude that the electron injection into the conduction bands of SWCNTs, on one hand, and the population of the ground state, on the other hand, renders to be faster in the films than in solution. Likewise, the kinetics, by which the 1003 and $1161 \mathrm{~nm}$ minima in 1a/SWCNTs convert upon photoexcitation to 999 and $1059 \mathrm{~nm}$ minima and to the baseline, are faster than in suspension. Here, the corresponding lifetimes are 40 and 4 ps.

Finally, we noted for 1a/ $\mathrm{C}_{60} @$ SWCNTs the immediate formation of a $1260 \mathrm{~nm}$ maximum and minima at 1019 and $1465 \mathrm{~nm}$ in the near infrared - Fig. 11. ${ }^{29}$ Again, these features transform with a few ps into blue shifted maxima and minima. Most dominant is the $1250 \mathrm{~nm}$ maximum and the $1004 \mathrm{~nm}$ minimum, whose formation dynamics give rise to 5 ps lifetime. The latter correlate with the electron injection into the conduction bands of SWCNTs. Once formed, its decay sets in with a $160 \mathrm{ps}$ lifetime to yield the ground state. ${ }^{30}$

\section{Conclusions}

Two Pc containing oligomers, that is, PPV-Zn(II)Pc 1a and PPV-Pd(II)Pc 1b as excited state electron donors have been synthesized and probed in terms of immobilization onto SWCNTs and $\mathrm{C}_{60} @$ @WCNTs. Importantly, the latter was chosen owing to the intrinsic charge transfer from SWCNTs to encapsulated $\mathrm{C}_{60}$. Such a charge transfer facilitates the immobilization of $\mathbf{1 a}$ and $\mathbf{1 b}$ and, in turn, the individualization of not only $\mathrm{C}_{60} @$ @WCNTs but also SWCNTs. Notably is that the larger radii of $\mathrm{C}_{60} @$ SWCNTs, when compared to SWCNTs, renders the stability of the resulting $\mathrm{C}_{60} @ S W C N T$ suspensions rather difficult. Still, sizeable electronic communications were found in absorption and fluorescence assays, which were complemented by Raman studies. Pump probe experiments on the femto- and picosecond time scales corroborated finally that the ground state charge transfer leads upon photoexcitation to the electron injection into conduction bands of SWCNTs and $\mathrm{C}_{60} @$ SWCNTs. 


\section{Acknowledgements}

Financial support is acknowledged to the Spanish MEC and MICINN (CTQ201124187/BQU and PRI-PIBUS-2011-1128). Generous funding by the DFG is greatly appreciated.

\section{References and Notes}

1 D. M. Guldi and N. Martín, Carbon Nanotubes and Related Structures, WileyVCH, 2010.

2 G. Bottari, G. de la Torre, D. M. Guldi and T. Torres, Chem. Rev., 2010, 110, 6768-6816.

3 R. Zhang, Y. Zhang, Q. Zhang, H. Xie, W. Qian and F. Wei, ACS Nano, 2013, 7, 6156-6161.

4 V. Sgobba and D. M. Guldi, Chem. Soc. Rev., 2009, 38, 165-184.

5 X. Wang, N. Behabtu, C. C. Young, D. E. Tsentalovich, M. Pasquali and J. Kono, Adv. Funct. Mater., 2014, DOI: 10.1002/adfm.201303865.

6 S. M. Bachilo, M. S. Strano, C. Kittrell, R. H. Hauge, R. E. Smalley and R. B. Weisman, Science, 2002, 298, 2361-2366.

7 G. M. A. Rahman, A. Troeger, V. Sgobba, D. M. Guldi, N. Jux, M. N. Tchoul, W. T. Ford, A. Mateo-Alonso and M. Prato, Chem. - Eur. J., 2008, 14, 8837-8846.

8 K. Dirian, M. Á. Herranz, G. Katsukis, J. Malig, L. Rodríguez-Pérez, C. RomeroNieto, V. Strauss, N. Martín and D. M. Guldi, Chem. Sci., 2013, 4, 4335-4353.

9 Z. Wang, S. Mohammadzadeh, T. Schmaltz, J. Kirschner, A. Khassanov, S. Eigler, U. Mundloch, C. Backes, H.-G. Steinrück, A. Magerl, F. Hauke, A. Hirsch and M. Halik, ACS Nano, 2013, 7, 11427-11434.

10 C. Oelsner, M. A. Herrero, C. Ehli, M. Prato and D. M. Guldi, J. Am. Chem. Soc., 2011, 133, 18696-18706.

11 J. Prasek, J. Drbohlavova, J. Chomoucka, J. Hubalek, O. Jasek, V. Adam and R. Kizek, J. Mater. Chem., 2011, 21, 15872-15884.

12 C. Romero-Nieto, R. García, M. Á. Herranz, L. Rodríguez-Pérez, M. SánchezNavarro, J. Rojo, N. Martín and D. M. Guldi, Angew. Chem., Int. Ed., 2013, 52, 10216-10220.

13 C. Romero-Nieto, R. García, M. Á. Herranz, C. Ehli, M. Ruppert, A. Hirsch, D. M. Guldi and N. Martín, J. Am. Chem. Soc., 2012, 134, 9183-9192.

14 D. A. Britz and A. N. Khlobystov, Chem. Soc. Rev., 2006, 35, 637-659.

15 L. Kavan, L. Dunsch and H. Kataura, Carbon, 2004, 42, 1011-1019.

16 J. Bartelmess, C. Ehli, J.-J. Cid, M. García-Iglesias, P. Vázquez, T. T. Torres, D. M. Guldi, M. Garcia-Iglesias and P. Vazquez, Chem. Sci., 2011, 2, 652-660.

17 Z. Li, V. Saini, E. Dervishi, V. P. Kunets, J. Zhang, Y. Xu, A. R. Biris, G. J. Salamo and A. S. Biris, Appl. Phys. Lett., 2010, 96, 033110.

18 J.-J. Cid, C. Ehli, C. Atienza-Castellanos, A. Gouloumis, E.-M. Maya, P. Vázquez, T. Torres and D. M. Guldi, Dalton Trans., 2009, 3955-3963.

19 T. Ahn, M. S. Jang, H.-K. Shim, D.-H. Hwang and T. Zyung, Macromolecules, 1999, 32, 3279-3285.

20 N. S. Cho, D.-H. Hwang, J.-I. Lee, B.-J. Jung and H.-K. Shim, Macromolecules, 2002, 35, 1224-1228.

21 L. Brinkhaus, G. Katsukis, J. Malig, R. D. Costa, M. Garcia-Iglesias, P. Vázquez, T. Torres and D. M. Guldi, Small, 2013, 9, 2348-2357. 
22 J. Malig, N. Jux, D. Kiessling, J.-J. Cid, P. Vázquez, T. Torres and D. M. Guldi, Angew. Chem., Int. Ed., 2011, 50, 3561-3565.

23 The small peak at $1923 \mathrm{~nm}$ is due to an artefact stemming from minor amounts of decomposed DMF during ultrasonication. This peak increases with prolonged ultrasonication.

24 A. G. S. Filho, A. Jorio, G. G. Samsonidze, G. Dresselhaus, R. Saito and M. S. Dresselhaus, Nanotechnology, 2003, 14, 1130-1139.

25 Y. F. Wang, X. W. Cao, S. F. Hu, Y. Y. Liu and G. X. Lan, Chem. Phys. Lett., 2001, 336, 47-52.

26 M. Kalbac, L. Kavan, M. Zukalova and L. Dunsch, J. Phys. Chem. B, 2004, 108, 6275-6280.

27 The lack of long term stability for 1b/ $\mathrm{C}_{60} @$ SWCNTs and 1a/ $\mathrm{C}_{60} @ S W C N T s$ hampered any meaningful analysis, besides that the fact the intrinsic bleaching in the near infrared region mirrors the ground state absorption.

28 The visible lacks notable transient absorption features.

29 The lack of long term stability for $\mathbf{1 b} / \mathbf{C}_{60} @ S W C N T s$ hampered any meaningful analysis, besides that the fact the intrinsic bleaching in the near infrared region mirrors the ground state absorption.

30 Individualized SWCNTs/ $\mathrm{C}_{60} @ S W C N T s$ are more soluble than small bundles and, in turn, are more likely to remain in solution. 